\title{
STOKES MULTIPLIERS FOR ASYMPTOTIC SOLUTIONS OF A CERTAIN DIFFERENTIAL EQUATION
}

\author{
BY \\ H. L. TURRITTIN
}

1. Introduction. The differential equation

$$
\frac{d^{n} y}{d x^{n}}-x^{v} y=0 \quad(n \geqq 2),
$$

where $v$ is a positive integer, has $n$ independent solutions

$$
y_{j}(x)=X^{j-1} \sum_{k=0}^{\infty} X^{p k} g(k p+j-1) \quad(j=1, \cdots, n)
$$

where $p=n+v, X=x p^{-n / p}$, and $g(w)$ is the reciprocal of a product of gamma functions,

$$
g(w)=1 / \prod_{k=1}^{n} \Gamma\left(\frac{w+p+1-k}{p}\right) .
$$

Series (2) converge for $|x|<\infty$ and are useful for computing the $y_{j}$ 's when $x$ is small. When $x$ is large, convergence is slow and it is more advantageous to use asymptotic expansions.

If we let $x=r e^{i \theta}$ and divide the $x$-plane into $2 p$ sectors by the radial lines $\theta=\pi h / p ; h=0,1, \cdots, 2 p-1$, then, as Trjitzinsky [8](1) has shown, there is associated with each of these sectors $n$ independent solutions $\bar{y}_{\mu}(x)$ of (1) such that $\bar{y}_{\mu}(x) \sim A_{\mu}(x)$ where the asymptotic forms

$$
A_{\mu}(x)=C Y_{\mu}^{1-t} \exp \left\{Y_{\mu}\right\} \sum_{m=0}^{\infty} c_{m} Y_{\mu}^{-m}
$$

where

$$
\begin{gathered}
t=\frac{1}{2}+\frac{n}{2}-\frac{n(n-1)}{2 p} ; \quad C=n^{t-3 / 2}(2 \pi)^{(1-n) / 2} \\
Y_{\mu}=(n / p) x^{p / n} \exp \{2 \pi i \mu / n\} ; \quad \text { and } \quad c_{0}=1
\end{gathered}
$$

The remaining $c_{m}$ 's are determined by formally substituting the expansions (4) into (1) and equating coefficients of successive powers of $x$ to zero. The subscript $\mu$ in (4) takes on $n$ successive integral values ranging from $-N$ to $P$ where $P=N=(n-1) / 2, n$ odd; and $P=n / 2$ and $N=P-1, n$ even. These

Presented to the Society, November 29, 1946; received by the editors September 3, 1949. (1) Numbers in brackets refer to the references cited at the end of the paper. 
asymptotic representations are valid uniformly throughout the particular sector under consideration, edges included.

Moreover there must exist a nonsingular linear relationship

$$
y_{j}(x)=\sum_{\mu=-N}^{P} D_{j \mu} \bar{y}_{\mu}(x) \quad(j=1, \cdots, n)
$$

between the $y_{j}$ 's and $\bar{y}_{\mu}$ 's. These constants $D_{j \mu}$, which change from sector to sector, are the Stokes multipliers. Unless the exact values of these multipliers can be found, the behavior of a given solution, defined by some specific linear combination of the $y_{j}$ 's, will be unknown when $x$ is large.

In this paper a method is given for computing these multipliers. The author has borrowed heavily from the Wright [9](2)-Ford [2]-Newsom [6]-Hughes [4] theory of asymptotic expansions. This WFNH-theory does not yield directly the desired uniform asymptotic representation in all cases, nor even the desired forms $A_{\mu}(x)$ when the real part of $Y_{\mu}$ is negative. The WFNH-theory is extended to supply the missing information. Theorems II and III contain the chief contributions in this direction, see $\$ 9$.

When $n>2$ the Stokes multipliers are not all uniquely determined in the $2 p$ sectors previously described; that is, in such a sector different solutions of (1) may have the same asymptotic expansion. If the sectors are properly enlarged, as will be later described, uniqueness of the multipliers can be restored.

Scheffé [7] computed two of the $n$ multipliers corresponding to each $j$.

The fundamental ideas presented here can be used to calculate multipliers for many other differential equations.

2. Reduction of the analysis to a single sector. Once the Stokes multipliers are known for a particular sector, those for other sectors can be readily computed, as will now be indicated. Suppose that $n$-independent solutions $\tilde{y}_{\mu}$ of (1) are known and that in sector

$$
S:-\pi<\alpha \leqq \arg (x) \leqq \beta<\pi
$$

the

$$
\tilde{y}_{\mu}(x) \sim A_{\mu}(x) \quad(\mu=-N, \cdots, P)
$$

and that

$$
y_{i}(x)=\sum_{\mu=-N}^{P} C_{j \mu} \tilde{y}_{\mu}(x) \quad(j=1, \cdots, n),
$$

where the multipliers $C_{j \mu}$ are also known; and that we are particularly interested in a new sector

$$
S^{\prime}: \alpha+2 \pi \zeta / p \leqq \arg (x) \leqq \beta+2 \pi \zeta / p
$$

(2) See also [10] for other references. 
where $\zeta=m n+\tau$ with $m$ and $\tau$ integers, $0 \leqq \tau<n$.

If this be the case, let $x^{\prime}=x \omega^{5}$ where $\omega=\exp \{2 \pi i / p\}$, then when $x$ is in $S, x^{\prime}$ is in $S^{\prime}$, and

$$
A_{\mu}\left(x^{\prime}\right)=A_{\mu+\zeta}(x)
$$

Also for any integer $k$

$$
A_{k+n}(x)=\omega^{v(1-n) / 2} A_{k}(x)
$$

By (9) and (10)

$$
A_{\mu}\left(x^{\prime}\right)=\omega^{m v(1-n) / 2} A_{\mu+\tau}(x)
$$

when $\mu=-N,-N+1, \cdots, P-\tau$ and

$$
A_{\mu}\left(x^{\prime}\right)=\omega^{v(1-n)(m+1) / 2} A_{\mu+\tau-n}(x)
$$

when $\mu=P-\tau+1, \cdots, P$ and $\tau>0$.

A set of $n$-independent solutions of (1), the $y_{\mu}^{\prime}(x)$ 's which have the prescribed asymptotic representations $A_{\mu}(x)$ in $S^{\prime}$, can now be specified as follows:

$$
y_{\mu}^{\prime}\left(x^{\prime}\right)=\omega^{m v(1-n) / 2} \tilde{y}_{\mu+\tau}(x) \quad \text { when } \mu=-N, \cdots, P-\tau ;
$$

and, if $\tau>0$,

$$
y_{\mu}^{\prime}\left(x^{\prime}\right)=\omega^{(m+1)(1-n) v / 2} \tilde{y}_{\mu+\tau-n}(x) \quad \text { when } \mu=P-\tau+1, \cdots, P .
$$

As a consequence of (7), (11), and (12)

$$
y_{\mu}^{\prime}(x) \sim A_{\mu}(x) \quad \text { if } x \text { is in } S^{\prime} .
$$

To obtain corresponding multipliers, first compute the original inverse multipliers $c_{\mu j}$ by solving system (8) for the $\tilde{y}_{\mu}$ 's, that is,

$$
\tilde{y}_{\mu}(x)=\sum_{j=1}^{n} c_{\mu j} y_{j}(x) \quad(\mu=-N, \cdots, P) .
$$

Next observe that

$$
y_{j}(x)=\omega^{5(1-j)} y_{j}\left(x^{\prime}\right) .
$$

Substituting (16) and (15) and the result into (13) and (14),

$$
y_{\mu}^{\prime}\left(x^{\prime}\right)=\omega^{m v(1-n) / 2} \sum_{j=1}^{n} c_{\mu+\tau, j} \omega^{5(1-j)} y_{j}\left(x^{\prime}\right)
$$

when $\mu=-N, \cdots, P-\tau$; and

$$
y_{\mu}^{\prime}\left(x^{\prime}\right)=\omega^{v(1-n)(m+1) / 2} \sum_{j=1}^{n} c_{\mu+\tau-n, j} \omega^{5(1-j)} y_{j}\left(x^{\prime}\right)
$$


when $\mu=P-\tau+1, \cdots, P$ and $\tau>0$.

The new inverse multipliers for sector $S^{\prime}$ are in evidence in (17) and (18) and the desired Stokes multipliers are found by solving (17) and (18) for the $y_{j}\left(x^{\prime}\right)$ 's. In this way multipliers for any sector $S^{\prime}$ can be computed by solving simultaneous linear equations, once the multipliers are known for some particular sector. Hence without loss of generality in the subsequent analysis, restrict the argument of $x$ to a particular sector called the $W$-sector, defined by $|\arg (x)| \leqq \pi n / 2 p, n$ odd; $-\pi(n+1) / 2 p \leqq \arg (x) \leqq \pi(n-1) / 2 p$, $n$ even.

Various subsectors of $W$ will also be considered; in particular let the $w$-sector be defined by $|\arg (x)| \leqq \pi / p, n$ odd; and $-3 \pi / 2 p \leqq \arg (x) \leqq \pi / 2 p$, $n$ 'even.

\section{Stokes multipliers for sector $w$.}

THEOREM I. If in system (6)

$$
D_{j \mu}=\omega^{\mu(1-j)} \quad \text { where } \omega=\exp \{2 \pi i / p\},
$$

then in sector w

$$
\bar{y}_{\mu}(x) \sim A_{\mu}(x) \quad(\mu=-N, \cdots, P) .
$$

To prove this theorem the behavior of the $\bar{y}_{\mu}$ 's will be studied not only in $w$, but also in the larger sector $W$, so that later uniqueness of asymptotic representation can be investigated. Begin by solving system (6) for the $\bar{y}_{\mu}$ 's and using Heineman's [3] work,

$$
\bar{y}_{\mu}(x)=-Q_{\mu} \sum_{j=1}^{n}(-1)^{i} E_{n-i, \mu} y_{j}(x) \quad(\mu=-N, \cdots, P)
$$

where

$$
Q_{\mu}=1 / \prod_{k=0}^{n-1}\left(\omega^{N-k}-\omega^{-\mu}\right)^{*} .
$$

In (21), as well as later in (27), the * signifies that the zero factor is to be deleted when the product is formed. In (21) this factor corresponds to $k=N+\mu$. The symbol $E_{n-j, \mu}$ represents the $(n-j)$ th elementary symmetric function formed from the arguments $\omega^{N}, \omega^{N-1}, \cdots, \omega^{-P}$ after $\omega^{-\mu}$ has been deleted from the list. For instance $E_{0 \mu}=1$,

$$
\begin{aligned}
E_{1, \mu} & =\omega^{N}+\cdots+\omega^{-\mu+1}+\omega^{-\mu-1}+\cdots+\omega^{-P}, \text { and } \\
E_{n-1, \mu} & =\omega^{N} \omega^{N-1} \cdots \omega^{-\mu+1} \omega^{-\mu-1} \cdots \omega^{-P} .
\end{aligned}
$$

If the series in (2) are substituted into (20), each $\bar{y}_{\mu}$ becomes the sum of $n$ absolutely convergent series. This sum can however be written as a single series of the form 


$$
\bar{y}_{\mu}(x)=-Q_{\mu} \sum_{m=0}^{\infty} C_{\mu}(m) X^{m} g(m)
$$

where the function

$$
C_{\mu}(M)=\frac{1}{p} \sum_{j=1}^{n} \sum_{k=1}^{p}(-1)^{j} E_{n-i, \mu} \omega^{k(M+1-j)} .
$$

To verify (23) observe that the sum $\sum_{k=1}^{p} \omega^{M k}$ equals $p$ if $M$ is an integral multiple of $p$, and equals 0 if $M$ is an integer which is not an integral multiple of $p$. Thus in effect (23) is the sum of the previously mentioned $n$ series.

To apply the WFNH-theory conveniently the terms in (23) will be regrouped. For this purpose introduce the constants

$$
G_{\mu k}=\omega^{k} \sum_{j=1}^{n}(-1)^{i} E_{n-j, \mu} \omega^{-k j} \quad(\mu=-N, \cdots, P) .
$$

In terms of the $G_{\mu k}$ 's

$$
C_{\mu}(M)=\frac{1}{p} \sum_{k=1}^{p} G_{\mu k} \omega^{M k} .
$$

A number of the $G$ 's are zero; to see this write the equation

$$
\sum_{j=1}^{n}(-1)^{j} E_{n-j, \mu} \chi^{j}=0 .
$$

Because the $E$ 's are the symmetric functions specified in (22), the roots of equation (25) are $\chi=0, \omega^{N}, \omega^{N-1}, \cdots, \omega^{-\mu+1}, \omega^{-\mu-1}, \cdots, \omega^{-P}$. Consequently when $i$ is an integer and $k=-N, \cdots, P$,

$$
G_{\mu, i}=0 \quad \text { if } i \equiv k \cdot(\bmod p) \text { unless } \mu \equiv i(\bmod p) .
$$

Note that $G_{\mu, i}=G_{\mu, k}$ if $i \equiv k(\bmod p)$.

Incidently $G_{\mu \mu}$ can be factored, thus

$$
G_{\mu \mu}=(-1)^{n} \prod_{j=0}^{n-1}\left(\omega^{-\mu}-\omega^{N-j}\right)^{*}
$$

see (21); and hence

$$
Q_{\mu} G_{\mu \mu}=-1 ; \quad \text { likewise } Q_{P} G_{P, p-N-1}=(-1)^{n} \omega^{n(n-1) / 2} .
$$

Utilizing the properties of the $G$ 's given in (26), the $C_{\mu}(M)$ can be abbreviated as follows:

$$
C_{\mu}(M)=\frac{1}{p}\left\{\sum_{k=\gamma}^{\xi} G_{\mu k} \omega^{M k}+G_{\mu, \mu+\beta} \omega^{(\mu+\beta) M}\right\},
$$


where $\gamma=P+1 ; \xi=p-N-1 ; \beta=0$ if $1 \leqq \mu \leqq P ;$ and $\beta=p$ if $-N \leqq \mu \leqq 0$. Hence (23) becomes

$$
\bar{y}_{\mu}(x)=-\frac{Q_{\mu}}{p}\left\{\sum_{k=\gamma}^{\xi} G_{\mu k} \sum_{m=0}^{\infty}\left(\omega^{k} X\right)^{m} g(m)+G_{\mu \mu} \sum_{m=0}^{\infty}\left(\omega^{\mu} X\right)^{m} g(m)\right\} .
$$

Note that in (30) each of the $(p-n+1)$ convergent series is of the form

$$
F(Z)=\sum_{m=0}^{\infty} g(m) Z^{m}
$$

Moreover such series are directly amenable to expansion by the WFNHtheory. This expansion however is postponed to $\$ 5$ while certain preliminaries are worked out in $\$ 4$.

Furthermore for our purposes in $\$ 6$ the function $C_{\mu}(M)$, which is a polynomial in powers of $\omega^{M}$, must be factored. To do so first consider the situation when $\mu=1, \cdots, P$; then it is clear from (24) that the sum

$$
\sum_{k=1}^{p} \omega^{(1-m-j) k}
$$

vanishes when $m=1,2, \cdots, p-n$ and $j=1, \cdots, n$. Consequently the polynomial $C_{\mu}(M)$ vanishes for $M=-1,-2, \cdots, n-p$. Likewise when $\mu=-N,-N+1, \cdots, 0, C_{\mu}(M)$ vanishes when $M=-1,-2, \cdots, n-p$.

Thus when $\mu=1, \cdots, P$ the polynomial $C_{\mu}(M)$ can be factored as follows:

$$
C_{\mu}(M)=-\frac{\omega^{\mu M} G_{\mu \mu}}{p}\left[\prod_{k=1}^{p-n}\left(\omega^{M}-\omega^{-h}\right)\right]\left[\sum_{k=0}^{P-\mu} L_{n-k} \omega^{M k}\right],
$$

where the constants $L_{k}=(-1)^{k} E_{k}$ and the $E_{k}$ are the elementary symmetric functions of $\omega^{0}, \omega^{1}, \cdots, \omega^{n-1}$. In particular $E_{0}=1 ; E_{1}=\omega^{0}+\omega^{1}+\cdots+\omega^{n-1}$; and $E_{n}=\omega^{0} \omega^{1} \cdots \omega^{n-1}=\omega^{n(n-1) / 2}$. Note also that

and

$$
L_{n-P+\mu}=-G_{\mu, p-N-1} / G_{\mu \mu}
$$

$$
L_{n}=(-1)^{n} \omega^{n(n-1) / 2} \quad \text { if } \mu \neq P .
$$

Similarly when $\mu=-N,-N+1, \cdots, 0$

$$
C_{\mu}(M)=\frac{\omega^{(P+1) M} G_{\mu \mu}}{p}\left[\prod_{h=1}^{p-n}\left(\omega^{M}-\omega^{-h}\right)\right]\left[\sum_{k=0}^{N+\mu} L_{k} \omega^{(N+\mu-k) M}\right] .
$$

In particular

$$
L_{0}=1 \text { and } L_{N+\mu}=G_{\mu, P+1}(-1)^{n+1} \omega^{n(n-1) / 2} / G_{\mu \mu}
$$

if $\mu \neq-N$. 
4. An expansion for $g(w)$. Two new functions are introduced:

$$
\Omega_{1}(w)=\Gamma(w) g\left(\frac{p w-p t}{n}\right) /(2 \pi)^{(1-n) / 2} n^{w-1 / 2}
$$

and

$$
\Omega_{2}(w)=(2 \pi)^{(1-n) / 2} n^{w-1 / 2} \prod_{h=0}^{n-1} \Gamma\left(\frac{h}{p}+\frac{t-1+w}{n}\right) / \Gamma(w)
$$

where $\Omega_{2}(w)=1 / \Omega_{1}(w)$. The function $g(w)$ which appears in (33) was defined in (3) and is a single-valued analytic function of $w$ throughout the entire finite w-plane.

Using the well known asymptotic expansion for $\log \Gamma(w)$ and proceeding as in $F\left({ }^{3}\right)$, pp. 74-75, an asymptotic expansion for $\Omega_{1}(w)$ is formed; namely

$$
\Omega_{1}(w)=1+\frac{h_{1}}{w}+\frac{h_{2}}{w^{2}}+\cdots+\frac{h_{q}+h(w, q)}{w^{q}} \quad(q=1,2,3, \cdots),
$$

where $h_{1}, h_{2}, \cdots$ are a definite set of constants and the function $h(w, q) \rightarrow 0$ uniformly as $w \longrightarrow \infty$ in any fixed closed sector $H_{-}$not containing the negative axis of the $w$-plane. Furthermore $h(w, q)$ is single-valued and analytic in $w$ except possibly for poles of the first order at $w=-1,-2, \cdots$.

Likewise an asymptotic expansion for $\Omega_{2}(1-w)$ can be found which takes the form

$$
\Omega_{2}(1-w)=1+\frac{\tilde{h}_{1}}{w}+\frac{\tilde{h}_{2}}{w^{2}}+\cdots+\frac{\tilde{h}_{q}+\tilde{h}(w, q)}{w^{q}} \quad(q=1,2, \cdots),
$$

where $\tilde{h}_{1}, \tilde{h}_{2}, \cdots$ are a definite set of constants and the function $\tilde{h}(w, q) \rightarrow()$ uniformly as $w \rightarrow \infty$ in any fixed closed sector $H_{+}$not containing the positive axis of the $w$-plane. Furthermore $\tilde{h}(w, q)$ is single-valued and analytic in $w$ except possibly for poles of the first order at $w=n m+t+k n / p$ where $k=0,1, \cdots, n-1 ; m=0,1,2, \cdots$.

Actually $\tilde{h}_{j}=h_{j}$, for $j=1,2, \cdots$; to prove this use the identity

$$
\Gamma(w) \Gamma(1-w)=2 \pi i /\left(e^{\pi i w}-e^{-\pi i w}\right)=\pi / \sin (\pi w)
$$

to see that

$$
\frac{\Omega_{1}(w)}{\Omega_{2}(1-w)}=\frac{\prod_{k=1}^{n}[1-\exp \{-(2 \pi i / n)(w-t+[n-n k] / p)\}]}{1-\exp \{-2 \pi i w\}} .
$$

Next observe that in the sector $-\pi<\arg (w)<0$

(3) Throughout this paper F signifies Ford [2]. 


$$
\begin{aligned}
\exp \{-2 \pi i w\} & \sim 0+\frac{0}{w}+\frac{0}{w^{2}}+\cdots ; \\
\exp \{-2 \pi i w / n\} & \sim 0+\frac{0}{w}+\frac{0}{w^{2}}+\cdots ;
\end{aligned}
$$

and because of (38)

$$
\Omega_{1}(w) / \Omega_{2}(1-w) \sim 1+\frac{0}{w}+\frac{0}{w^{2}}+\cdots ;
$$

hence $\tilde{h}_{j}=h_{j}, j=1,2, \cdots$.

Expansion (35) may be transformed into

$$
\begin{array}{r}
\Omega_{1}(w)=1+\frac{c_{1}}{w}+\frac{c_{2}}{w(w+1)}+\cdots+\frac{c_{q}+c(w, q)}{w(w+1) \cdots(w+q-1)} \\
(q=1,2, \cdots),
\end{array}
$$

where $c_{1}, c_{2}, \cdots$ are an appropriate set of constants and the function $c(w, q) \rightarrow 0$ uniformly as $w \rightarrow \infty$ on any ray located in sector $H_{-}$. The $c(w, q)$ is a single-valued analytic function of $w$ except possibly for poles of the first order at $w=-q,-q-1,-q-2, \cdots$.

The $c(w, q)$ also satisfies the two inequalities

$$
|c(w, q)|<\frac{K_{q}}{|w|} \text { and }\left|\frac{d c(w, q)}{d w}\right|<\frac{K_{q}}{|w|^{2}}
$$

if $w$ is in $H_{-},|w| \geqq 1$, and $K_{q}$ is an appropriate constant. These inequalities can be verified by proceeding as in $\mathrm{F}$, pp. 80-81.

Similarly (36) can be converted into

$$
\begin{array}{r}
\Omega_{2}(1-w)=1+\frac{c_{1}}{w}+\frac{c_{2}}{w(w+1)}+\cdots+\frac{c_{q}+\tilde{c}(w, q)}{w(w+1) \cdots(w+q-1)} \\
(q=1,2, \cdots),
\end{array}
$$

where the $c_{1}, c_{2}, \cdots$ are not only the same as those appearing in (39), but also, as we shall see, the same constants as those appearing in (4). The function $\tilde{c}(w, q) \rightarrow 0$ uniformly as $w \rightarrow \infty$ in sector $H_{+}$. The $\tilde{c}(w, q)$ is a singlevalued analytic function of $w$ except possibly for poles of the first order at $w=n m+t+k n / p$ where $k=0,1, \cdots, n-1$; and $m=0,1,2, \cdots$. Moreover if $w$ is in the sector $H_{+}$

$$
|\tilde{c}(w, q)|<K_{q} /|w| \text { and }\left|\frac{d \tilde{c}(w, q)}{d w}\right|<K_{q} /|w|^{2} \quad(|w|>1)
$$

where $K_{q}$ is an appropriate constant; again see F, pp. 80-81. 
As a consequence of (39)

$$
q\left(\frac{p w-p t}{n}\right)=C n^{w+1-t}\left[\frac{1}{\Gamma(w)}+\frac{c_{1}}{\Gamma(w+1)}+\cdots+\frac{c_{q}+c(w, q)}{\Gamma(w+q)}\right]
$$

where $q=1,2, \cdots$ and $C$ is given in (5); compare this result with Hughes [5, p. 457]. This is the desired expansion for $g(w)$ in sector $H_{-}$.

5. An application of the WFNH-theory. The WFNH-theory will now be applied to the functions of type (31) which appear in (30). When the bounds for the reciprocal of a gamma function given by $F, \mathrm{pp} .61-62$, are used, it is found that, if an $\epsilon$ is chosen and $x_{0}$ is an arbitrarily fixed large negative number, then

$$
|g(x+i y)|<K \exp \{|y|(\epsilon+n \pi / 2 p)\}
$$

for all $x>x_{0}$ and for any $y$ provided that the point $w=x+i y$ is at least one unit distant from the negative axis of the $w$-plane. The constant $K$ depends only upon $\epsilon$ and $x_{0}$.

F's first theorem, p. 5, as modified by his note (c), p. 11, applies to (31); hence, if $\pi n / 2 p<\arg (Z)<2 \pi-n \pi / 2 p$, then

$$
\sum_{m=0}^{\infty} g(m) Z^{m}=-\sum_{m=1}^{L} g(-m) Z^{-m}+\eta_{L}(Z)
$$

where

$$
\eta_{L}(Z)=\frac{i}{2} \int_{-L-\alpha}\left[g(s)\left(e^{-\pi i} Z\right)^{s} / \sin \pi s\right] d s
$$

where $L=1,2, \cdots$ and $\alpha$ a constant, $0<\alpha<1$. The path of integration in (43) is a vertical line, $\Re[s]=-L-\alpha$, in the $s$-plane with integration up the line. A more common notation for such an integration would have been $\int_{-L-\alpha-i \infty}^{-L-\alpha+\infty}$. Also if

$$
\epsilon+\pi n / 2 p \leqq \arg (Z) \leqq 2 \pi-\epsilon-n \pi / 2 p
$$

where $\epsilon$ is an arbitrarily small fixed positive number, then uniformly in sector (44) the

$$
\lim _{Z \rightarrow \infty} Z^{L} \eta_{L}(Z)=0
$$

If $x$ is in sector $W$ and $Z=\omega^{k} X$ with $k=P+1, \cdots, p-N-1$, inequality (44) is satisfied. Therefore F's first theorem yields an asymptotic expansion in $W$ for each of the first $(p-n)$ infinite series in (30).

To get an expansion for the one remaining series in (30), consider a sector $H_{-}$in the $Z$-plane defined by $|\arg (Z)| \leqq \pi-\epsilon$; then if $F(Z)$ is defined by (31) and $Z$ is in $H_{-}$, F's second theorem, pp. $30-31$, states that 


$$
F(Z)=\int_{-L-\alpha}^{\infty} g(s) Z^{s} d s-\sum_{m=1}^{L} g(-m) Z^{-m}+\delta_{1}(Z, L)
$$

where $L=1,2, \cdots$ and $\alpha$ is a constant, $0<\alpha<1$. Moreover as $Z \rightarrow \infty$ in $H_{-}$ uniformly $Z^{L} \delta_{1}(Z, L) \rightarrow 0$.

To procure a complete asymptotic representation of $F(Z)$ in $H_{-}$, an expansion for the integral in (45) is needed. To get it note that because of (41)

$$
\begin{aligned}
& \int_{-L-\alpha}^{\infty} g(s) Z^{s} d s \\
& \quad=p C \int_{-\lambda}^{\infty}\left(n Z^{p / n}\right)^{w+1-t}\left[\frac{1}{\Gamma(w+1)}+\cdots+\frac{c_{q}+c(w+1, q)}{\Gamma(w+q+1)}\right] d w,
\end{aligned}
$$

where $\lambda=1-t+(L+\alpha) n / p, L=1,2, \cdots ; q=1,2, \cdots$. Then use Hughes's results $[4$, p. 426], and conclude from (46) that for values of $Z$ of large modulus such that $|\arg (Z)| \leqq \pi n / 2 p$,

$$
\begin{aligned}
& \int_{-L-\alpha}^{\infty} q(s) Z^{s} d s \\
& \quad=p C\left(n Z^{p / n}\right)^{1-t} \exp \left\{n Z^{p / n}\right\}\left[\sum_{m=0}^{q} c_{m}\left(n Z^{p / n}\right)^{-m}+\left(n Z^{p / n}\right)^{-q} \delta_{2}(Z, q, L)\right]
\end{aligned}
$$

when $L$ is sufficiently large, say $L=p(n+2 q)$.

If

$$
\left|\delta_{2}(Z, q, L)\right|<K
$$

a constant,

when $|\arg (Z)| \leqq n \pi / 2 p$ and $|Z|$ is large, the right member of (47) furnishes an asymptotic expansion for the integral on the left. Wright's [11] Theorem 5 guarantees the existence of this constant $K$. Wright's proofs are given in [9] and [12].

The existence of the $K$ in (48) may also be based on our Theorem III. This theorem combines F's two theorems, pp. 38 and 48, into a single theorem. Our proof is patterned after F, but due care is taken to show uniform convergence, which is lacking in F's work, for at the bottom of p. 43 F's $\sigma(\epsilon)$ is a function of both $\epsilon$ and $\phi$.

Substituting the expansions (42), (45), and (47) into (30) an elaborate expansion is found for $\bar{y}_{\mu}(x)$ in sector $W_{1 \mu}$, that portion of sector $W$ where the $\left|\arg \left(\omega^{\mu} X\right)\right| \leqq n \pi / 2 p$. Fortunately this expansion for $\bar{y}_{\mu}(x)$ can be simplified because

$$
\sum_{k=\gamma}^{\xi} G_{\mu k} \sum_{m=1}^{L} g(-m)\left(\omega^{k} X\right)^{-m}+G_{\mu \mu} \sum_{m=1}^{L} g(-m)\left(\omega^{\mu} X\right)^{-m} \equiv 0 .
$$

To verify (49) first note that the coefficient of $X^{-m}$ in (49) is 


$$
g(-m)\left[\sum_{k=\gamma}^{\xi} G_{\mu k \omega^{-k m}}+G_{\mu \mu} \omega^{-\mu m}\right]
$$

and this expression is zero if $m=p-j, 2 p-j, 3 p-j, \cdots$, where $j=0,1, \cdots$, $(n-1)$, for $g(-m)$ is zero for these values of $m$.

Also if $m$ is an integer by (29) and (24)

$$
\begin{aligned}
& \sum_{k=\gamma}^{\xi} G_{\mu k \omega^{-k m}}+G_{\mu \mu} \omega^{-\mu m}=p C_{\mu}(-m) \quad \text { and } \\
& p C_{\mu}(-m)=\sum_{j=1}^{n}(-1)^{j} E_{n-j, \mu} \sum_{k=1}^{p} \omega^{(-m+1-j) k} .
\end{aligned}
$$

The last member of (51) is zero when $m=L p+1, L p+2, \cdots, L p+p-n$ and $L=1,2, \cdots$, because the sums

$$
\sum_{k=1}^{p} \omega^{(1-m-j) k} \quad(j=1, \cdots, n)
$$

all vanish for these values of $m$. Thus when $m=1,2,3, \cdots,(50)$ vanishes and (49) is substantiated.

Utilizing (49), the expansion for $\bar{y}_{\mu}(x)$ in $W_{1 \mu}$ reduces to

$$
\begin{aligned}
\bar{y}_{\mu}(x)=\frac{-Q_{\mu}}{p} & \left\{\sum_{k=\gamma}^{\xi} G_{\mu k} \eta_{L}\left(\omega^{k} X\right)+G_{\mu \mu} \delta_{1}\left(\omega^{\mu} X, L\right)\right. \\
& \left.+G_{\mu \mu}\left[p C Y_{\mu}^{1-t} \exp \left\{Y_{\mu}\right\}\left(\sum_{m=0}^{q} c_{m} Y_{\mu}^{-m}+Y_{\mu}^{-q} \delta_{2}\left(\omega^{\mu} X, q\right)\right)\right]\right\}
\end{aligned}
$$

where

$$
L=(n+2 q) p \quad \text { and } \quad q=1,2, \cdots ; \quad \mu=-N, \cdots, P .
$$

In $W_{1 \mu}$ uniformly the

$$
\begin{array}{ll}
\lim _{X \rightarrow \infty} X^{L} \eta_{L}\left(\omega^{k} X\right)=0 & (k=P+1, \cdots, p-N-1) ; \\
\lim _{X \rightarrow \infty} X^{L} \delta_{1}\left(\omega^{\mu} X, L\right)=0 ;
\end{array}
$$

and

$$
\left|\delta_{2}\left(\omega^{\mu} X, q\right)\right|<K
$$

a constant.

Preserving only the dominant terms in (53) and recalling (28), in $W_{1 \mu}$

$$
\bar{y}_{\mu}(x) \sim C Y_{\mu}^{1-t} \exp \left\{Y_{\mu}\right\} \sum_{m=0}^{\infty} c_{m} Y_{\mu}^{-m} .
$$

This asymptotic form is precisely that given in (4). 
Expansion (55) is of course valid also in any portion of the w-sector where $\left|\arg \left(\omega^{\mu} X\right)\right| \leqq n \pi / 2 p$.

Let $W_{2 \mu}$ be that part of $W$ which remains after sector $W_{1 \mu}$ has been deleted from $W$. The WFNH-theory can also be applied to (30) in $W_{2 \mu}$, see Hughes $[4$, p. 426$]$, with the result that

$$
\bar{y}_{\mu}(x) \sim 0+\frac{0}{x}+\frac{0}{x^{2}}+\cdots .
$$

This result is certainly correct, but not sufficiently precise for our purposes. An asymptotic expansion is needed for $\bar{y}_{\mu}(x)$ which indicates how rapidly $\bar{y}_{\mu}(x) \rightarrow 0$ as $x \rightarrow \infty$. The determination of such an expansion requires a definite extension of the WFNH-theory. In the next section this problem is attacked.

6. An integral expression for $\bar{y}_{\mu}(x)$ in $W_{2 \mu}$. In computing an asymptotic expansion for $\bar{y}_{\mu}$ in $W_{2 \mu}$, one of the following four inequalities will specify the extent of $W_{2 \mu}$ :

(56) Case I: $\quad n$ odd, $\pi / 2<\arg \left(\omega^{\mu p / n} X^{p / n}\right) \leqq \pi / 2+2 \pi \mu / n ; \quad \mu=1, \cdots, P$;

(57) Case II: $n$ even, $\pi / 2<\arg \left(\omega^{\mu p / n} X^{p / n}\right) \leqq \pi(n-1) / 2 n+2 \pi \mu / n$;

$$
\mu=1, \cdots, P \text {; }
$$

(58) Case III: $n$ odd, $-\pi / 2+2 \pi \mu / n \leqq \arg \left(\omega^{\mu / n} X^{p / n}\right)<-\pi / 2$;

$$
\mu=-1,-2, \cdots,-N
$$

(59) Case IV: $n$ even, $-\pi(n+1) / 2 n+2 \pi \mu / n \leqq \arg \left(\omega^{\mu p / n} X^{p / n}\right)<-\pi / 2$;

$$
\mu=0,-1, \cdots,-N .
$$

Each of these cases requires slightly different treatment. The odd case when $\mu=0$ does not occur because (55) is then valid throughout $W$.

If $x$ is in $W_{2 \mu}$, F's first theorem, p. 5, can be applied to each of the sums in (30) and in the resulting expansion the terms involving $g(-m)$ all cancel out netting

$$
\bar{y}_{\mu}(x)=\frac{-Q_{\mu}}{p}\left\{\sum_{k=\gamma}^{\xi} G_{\mu k} \eta_{L}\left(\omega^{k} X\right)+G_{\mu \mu} \eta_{L}\left(\omega^{k+\beta} X\right)\right\},
$$

where from here on $\beta=0$ in cases I and II and $\beta=p$ in cases III and IV. In short the asymptotic expansions which usually occur in the WFNH-theory have all disappeared and only the remainder terms are left.

Utilizing (43) and (29), in $W_{2 \mu}$

$$
\bar{y}_{\mu}(x)=\frac{-i Q_{\mu}}{2 p} \int_{-L-\alpha} H_{\mu}(s, X) d s,
$$

where the function 


$$
H_{\mu}(s, X)=p g(s)\left(e^{-\pi i} X\right)^{8} C_{\mu}(s) / \sin (\pi s) .
$$

If an asymptotic expansion for $\bar{y}_{\mu}$ is to be found, it must be derived from the integral in (60). With this objective in mind the integrand will be transformed into a more tractable form.

We need the identity

$$
\sin (\pi s)=(-2)^{p-1} \prod_{h=0}^{p-1} \sin \left(\frac{\pi s-\pi h}{p}\right) .
$$

Using (37) it is easily shown that

$$
g(s)=(-\pi)^{-n} \prod_{h=0}^{n-1}\left[\Gamma\left(\frac{h-s}{p}\right) \sin \left(\frac{\pi s-\pi h}{p}\right)\right] .
$$

Since $\omega=\exp \{2 \pi i / p\}$,

$$
\prod_{h=1}^{p-n}\left(\omega^{M}-\omega^{-h}\right)=\left[-2 \omega^{(2 M+n-1) / 4}\right]^{p-n}\left[\prod_{k=n}^{p-1} \sin \left(\frac{\pi M-\pi k}{p}\right)\right] .
$$

Returning to the integrand of (60) and using (61), (62), (32), and (63), when $\mu=1, \cdots, P$

$$
H_{\mu}(s, X)=J_{1} G_{\mu \mu} X_{\mu}^{s}\left[\prod_{h=0}^{n-1} \Gamma\left(\frac{h-s}{p}\right)\right]\left[\sum_{k=0}^{P-\mu} L_{n-k} \omega^{k s}\right]
$$

where the constant

$$
J_{1}=-2 i \omega^{n(1-n) / 4}(-2 \pi i)^{-n}
$$

and

$$
X_{\mu}=e^{-\pi i} \omega^{\mu+(p-n) / 2} X .
$$

Similarly when $\mu=-N,-N+1, \cdots, 0$

$$
H_{\mu}(s, X)=-J_{1} G_{\mu \mu} X_{P+1}^{s}\left[\prod_{h=0}^{n-1} \Gamma\left(\frac{h-s}{p}\right)\right]\left[\sum_{k=0}^{N+\mu} L_{k} \omega^{s(N+\mu-k)}\right] .
$$

By virtue of (34), (64), and (65) when $\mu=1, \cdots, P$

$$
H_{\mu}(s, X)=(2 \pi)^{(n-1) / 2} J_{1} G_{\mu \mu} X_{\mu}^{s} n^{.5-S} \Omega_{2}(S) \Gamma(S) \sum_{k=0}^{P-\mu} L_{n-k} \omega^{s k}
$$

where $S=1-t-n s / p ;$ and when $\mu=-N,-N+1, \cdots, 0$

$$
H_{\mu}(s, X)=-(2 \pi)^{(n-1) / 2} J_{1} G_{\mu \mu} X_{P+1}^{8} n^{.5-S} \Omega_{2}(S) \Gamma(S) \sum_{k=0}^{N+\mu} L_{k} \omega^{*(N+\mu-k)}
$$

Using these expressions for the integrand, when $\mu=1, \cdots, P,(60)$ be- 
comes

$$
\bar{y}_{\mu}(x)=J_{2} \sum_{k=0}^{P-\mu} L_{n-k} \int_{-L-a} n^{n z / p} X_{\mu+k}^{8} \Omega_{2}(S) \Gamma(S) d s
$$

where $J_{2}=i n^{t-.5} J_{1} / 2 p(2 \pi)^{(1-n) / 2}$. Also when $\mu=-N,-N+1, \cdots, 0$

$$
\bar{y}_{\mu}(x)=-J_{2} \sum_{k=0}^{N+\mu} L_{k} \int_{-L-\alpha} n^{n s / p} X_{n-k+\mu}^{s} \Omega_{2}(S) \Gamma(S) d s .
$$

These are the desired integral expressions for $\bar{y}_{\mu}(x)$ in $W_{2 \mu}$. All the integrals in (66) and (67) are of the same type; namely

$$
I_{\sigma}(x)=\int_{-L-\alpha} Z_{\sigma}^{8} \Omega_{2}(S) \Gamma(S) d s
$$

where

$$
\sigma=1, \cdots, n \text { and } Z_{\sigma}=x(n / p)^{n / p} \omega^{\sigma-n / 2} .
$$

7. Asymptotic expansions for $\bar{y}_{\mu}$ in $W_{2 \mu}$. Use (40) and the identity $\Gamma(1+w)=w \Gamma(w)$ to get the expansion

$$
\Gamma(1-w) \Omega_{2}(1-w)=\sum_{j=0}^{q} c_{j}(-1)^{i} \Gamma(1-j-w)+(-1)^{q} \tilde{c}(w, q) \Gamma(1-q-w)
$$

with $q=1,2,3, \cdots$. Hence (68) becomes

$$
\begin{aligned}
I_{\sigma}(x)= & \sum_{j=0}^{q}(-1)^{j} c_{j} \int_{-L-\alpha} Z_{\sigma}^{s} \Gamma(S-j) d s \\
& +(-1)^{q} \int_{-L-\alpha} Z_{\sigma}^{s} \tilde{c}(t+n s / p, q) \Gamma(S-q) d s .
\end{aligned}
$$
[1]

$$
(1 / 2 \pi i) \int_{x-i \infty}^{x+i \infty} z^{-\imath} \Gamma(s) d s=e^{-z}
$$

provided (a) $x>0$, and (b) $|\arg (z)|<\pi / 2$. In order that in every case stipulation (a) is satisfied for the integrals of (70), $L$ must be sufficiently large relative to $q$. The choice of $L$ in (54) is amply large for this purpose. For (b) to be satisfied it is necessary that the $\left|\arg \left(Z_{\sigma}\right)\right|<n \pi / 2 p$. By referring to (56)(59), (69), (66), and (67), it is clear that $\left|\arg \left(Z_{\sigma}\right)\right|<n \pi / 2 p$ in $W_{2 \mu}$ for the $\sigma$ 's under consideration in (66) and (67).

After evaluation of the integrals of (70) by (71) and observing that $Z_{\sigma}^{p / n}=e^{-\pi i} Y_{\sigma}$, it is clear that 


$$
I_{\sigma}(x)=-i\left(\frac{2 \pi p}{n}\right) Y_{\sigma}^{1-t} \exp \left\{Y_{\sigma}+\pi i t\right\}\left[\sum_{j=0}^{q} c_{j} Y_{\sigma}^{-j}-R_{\sigma}(q, x) Z_{\sigma}^{-p q / n}\right]
$$

where

$$
\begin{aligned}
R_{\sigma}(q, x)= & (n i / 2 \pi p) Z_{\sigma}^{p(t-1+q) / n}(-1)^{q} \exp \left\{Z_{\sigma}^{p / n}\right\} \\
& \cdot \int_{-L-\alpha} Z_{\sigma}^{s} \tilde{c}(t+n s / p, q) \Gamma(S-q) d s .
\end{aligned}
$$

When Theorem II of $\S 9$ is applied to the integral in (72) it is found that $R_{\sigma}(q, x) \rightarrow 0$ uniformly as $x \rightarrow \infty$ on any ray in $W_{2 \mu}$. Therefore the following asymptotic expansion is valid in $W_{2 \mu}$ :

$$
I_{\sigma}(x) \sim(-2 \pi i p / n) Y_{\sigma}^{1-t} \exp \left\{Y_{\sigma}+\pi i t\right\} \sum_{j=0}^{\infty} c_{j} Y_{\sigma}^{-j}
$$

Because of (66), (67), and (68)

$$
\bar{y}_{\mu}(x)=J_{2} \sum_{k=0}^{P-\mu} L_{n-k} I_{\mu+k}(x) \quad(\mu=1, \cdots, P) ;
$$

and

$$
\bar{y}_{\mu}(x)=-J_{2} \sum_{k=0}^{N+\mu} L_{k} I_{n-k+\mu}(x) \quad(\mu=-N,-N+1, \cdots, 0) .
$$

Substitute the expansion in (73) into (74) and (75) to get expansions for the $\bar{y}_{\mu}$ 's. The resulting expressions will be, in general, the sum of several expansions. The particular expansion which is dominant in $W_{2 \mu}$ must now be selected. For this purpose set $x=\rho e^{i \phi}$. Then the relative magnitudes of the various $I_{\sigma}$ 's for large values of $\rho$ depend primarily upon the size of the respective exponential factors

$$
\left|\exp \left\{-Z_{\sigma}^{p / n}\right\}\right|=\exp \left\{(n / p) \rho^{p / n} \cos \left(\frac{p \phi+2 \pi \sigma}{n}\right)\right\} ;
$$

and these quantities in turn depend primarily upon the values of the corresponding quantities $\cos ((p \phi+2 \pi \sigma) / n)$. To pick the dominant expansion, draw the various cosine curves

$$
y=\cos \left(\frac{p \phi+2 \pi \sigma}{n}\right)
$$

with the $\phi$-axis as abscissa and the $y$-axis as ordinate; and compare the ordinates. If $\phi$ is restricted to the sector $w$, it is found that the dominant term in (74) and (75) corresponds in every case to $k=0$.

Thus in the portion of the $w$-sector common to $W_{2 \mu}$ 


$$
\begin{aligned}
\bar{y}_{\mu}(x) \sim i J_{2} L_{n}(-2 \pi p / n) Y_{\mu}^{1-t} \exp \left\{Y_{\mu}+\pi i t\right\} \sum_{j=0}^{\infty} c_{j} Y_{\mu}^{-j}= & A_{\mu}(x) \\
& (\mu=1, \cdots, P)
\end{aligned}
$$

and since $Y_{n+\mu}=Y_{\mu} e^{2 \pi i}$

$$
\begin{aligned}
\bar{y}_{\mu}(x) \sim i J_{2} L_{0}(2 \pi p / n) Y_{\mu}^{1-t} \exp \left\{Y_{\mu}-\pi i t\right\} \sum_{j=0}^{\infty} c_{j} Y_{\mu}^{-j} & =A_{\mu}(x) \\
(\mu & =-N, \cdots, 0) .
\end{aligned}
$$

This completes the proof of Theorem I and guaranties that the $D_{j \mu}$ 's given in (19) are the Stokes multipliers corresponding to the $\bar{y}_{\mu}(x)$ in the $w$-sector.

8. Uniqueness of Stokes multipliers. A glance at the cosine curves (76) makes it clear that, if $n \geqq 4, n$-independent solutions of (1) other than the $\bar{y}_{\mu}$ 's can be found which will have precisely the same asymptotic expansions $A_{\mu}(x)$ in $w$ as the $\bar{y}_{\mu}$ 's. For instance such an independent set can be formed by retaining all the $\bar{y}_{\mu}$ 's except $\bar{y}_{0}$ and replacing $\bar{y}_{0}$ by $\left(\bar{y}_{0}+\bar{y}_{P}\right)$. Thus in general the Stokes multipliers for the w-sector are not unique.

However by utilizing (73), (74), and (75) and more carefully examining the various cosine graphs (76), a way to reestablish the uniqueness of Stokes multipliers becomes evident. The w-sector is too narrow to give uniqueness. The entire sector $W$ should have been used instead and in place of the functions $\bar{y}_{\mu}(x)$ we should have used a different set of independent solutions, namely the $\tilde{y}_{\mu}(x)$ which will now be specified.

Let $M=P / 2$ if $P$ is even; and let $M=(P-1) / 2$ if $P$ is odd; then set

$$
\begin{array}{rlrl}
\tilde{y}_{\mu} & =J_{2} L_{n} I_{\mu} & (\mu=P-M, \cdots, P), \\
\tilde{y}_{\mu} & =J_{2} L_{n} I_{\mu}+J_{2} \sum_{k=1}^{P-2 \mu} L_{n-k} I_{\mu+k} & (\mu=1, \cdots, P-M-1 ; n>5), \\
\tilde{y}_{0} & =-J_{2} L_{0} I_{n}-J_{2} \sum_{k=1}^{N} L_{k} I_{n-k} & \\
\tilde{y}_{\mu} & =-J_{2} L_{0} I_{n+\mu}-J_{2} \sum_{k=1}^{N+2 \mu} L_{k} I_{n-k+\mu} & (n>2), \\
\tilde{y}_{-N+\mu}=-J_{2} L_{0} I_{n-N+\mu}, &
\end{array}
$$

where in the fourth equation $\mu=-1,-2, \cdots,(1+M-N)$ unless $n$ and $P$ are both even, in this event replace $(1+M-N)$ by $(M-N)$, and in the fifth equation $\mu=0,1, \cdots, M$ unless $n$ and $P$ are both even, in this event replace $M$ by $(M-1)$.

On examining the asymptotic behavior of $\tilde{y}_{\mu}$ it is found that in sector $W_{2 \mu}$ the $I$ appearing immediately to the right of the equality sign in (77) is 
dominant; therefore in $W_{2 \mu} \tilde{y}_{\mu}(x) \sim A_{\mu}(x)(\mu=-N, \cdots, P)$.

By virtue of (74), (75), and (77)

$$
\begin{array}{lr}
\bar{y}_{\mu}=L_{n}^{-1} \sum_{k=0}^{P-\mu} L_{n-k} \tilde{y}_{\mu+k} & (\mu=P-M, \cdots, P), \\
\bar{y}_{\mu}=\tilde{y}_{\mu}+L_{n}^{-1} \sum_{k=P-2 \mu+1}^{P-\mu} L_{n-k} \tilde{y}_{\mu+k} & (\mu=1, \cdots, P-M-1 ; n>5), \\
\bar{y}_{0}=\tilde{y}_{0}, & \quad(n>6), \\
\bar{y}_{\mu}=\tilde{y}_{\mu}+L_{0}^{-1} \sum_{k=N+2 \mu+1}^{N+\mu} L_{k} \tilde{y}_{-k+\mu} & \\
\bar{y}_{\mu}=L_{0}^{-1} \sum_{k=0}^{N+\mu} L_{k} \tilde{y}_{-k+\mu}, &
\end{array}
$$

where in the fourth equation $\mu=-1, \cdots,(1+M-N)$ unless $n$ and $P$ are both even, in this event replace $(1+M-N)$ by $(M-N)$, and in the fifth equation $\mu=-N, \cdots,(M-N)$ unless $n$ and $P$ are both even, in this event replace $(M-N)$ by $(M-N-1)$.

If system (78) is solved for the $\bar{y}_{\mu}$ 's;

$$
\begin{aligned}
\tilde{y}_{P}=\bar{y}_{P} ; \quad \tilde{y}_{0}=\bar{y}_{0} ; \quad \tilde{y}_{-N}=\bar{y}_{-N}, & \quad(j=1, \cdots, M), \\
\tilde{y}_{P-j}= & \bar{y}_{P-j}+\mathcal{L}\left(\bar{y}_{P-j+1}, \bar{y}_{P-j+2}, \cdots, \bar{y}_{P}\right) \\
\tilde{y}_{j}= & \bar{y}_{j}+\mathcal{L}\left(\bar{y}_{P+1-j}, \bar{y}_{P+2-j}, \cdots, \bar{y}_{P}\right) \\
& \quad(n>5 ; j=1, \cdots, P-M-1), \\
\tilde{y}_{-j}= & \bar{y}_{-j}+\mathcal{L}\left(\bar{y}_{-N+j-1}, \bar{y}_{-N+j-2}, \cdots, \bar{y}_{-N}\right), \\
\tilde{y}_{j-N}= & \bar{y}_{j-N}+\mathcal{L}\left(\bar{y}_{j-N-1}, \bar{y}_{j-N-2}, \cdots, \bar{y}_{-N}\right)
\end{aligned}
$$

where in the sixth equation $j=1, \cdots,(N-M-1) ; n>6$; unless $n$ and $P$ are both even, in this event replace $(N-M-1)$ by $(N-M)$, and in the seventh equation $j=1, \cdots, M$ unless $n$ and $P$ are both even, in this event replace $M$ by $(M-1)$.

In (79) the function $\mathcal{L}(\cdots)$ indicates an appropriate linear combination of its arguments; moreover in the expressions for $\tilde{y}_{\mu}$ the dominant $\bar{y}$ in $W_{1 \mu}$ is the $\bar{y}$ appearing immediately to the right of the equality sign. The $\bar{y}$ 's in the unspecified linear combination are all subdominant in $W_{1 \mu}$; therefore in $W_{1 \mu}, \tilde{y}_{\mu}(x) \sim A_{\mu}(x)(\mu=-N, \cdots, P)$ and hence throughout $W \tilde{y}_{\mu}(x) \sim A_{\mu}(x)$ $(\mu=-N, \cdots, P)$. Moreover it is clear from the cosine graphs that no other linear combination of solutions could have these same asymptotic expansions throughout the $W$-sector. The Stokes multipliers for the $\tilde{y}_{\mu}$ 's are therefore uniquely determined in $W$. 
To compute the multipliers corresponding to the $\tilde{y}_{\mu}$ 's, substitute the values of $\bar{y}_{\mu}$ given in (78) into (6), using the values of the $D_{j \mu}$ 's given in (19), and find the coefficients of the various $\tilde{y}_{\mu}$ 's. These coefficients will be the desired Stokes multipliers. The author was in hopes that these new multipliers would reduce to expressions comparable in simplicity with those for the $D_{j \mu}$ 's in (19); but he has not succeeded in making appreciable simplifications in the elaborate expressions for the multipliers corresponding to the $\tilde{y}_{\mu}$ 's. Uniqueness has therefore been attained at the expense of simplicity.

\section{Two fundamental theorems and a lemma.}

THEOREM II. If $P(w)$ is a single-valued analytic function of the complex variable $w=x+i y$ throughout the half-plane $x \geqq x_{0}$, where $x_{0}$ has some assigned negative value; if when $\Re[w] \geqq 0$

$$
|P(w)|<B /|w| \text { and }|d P(w) / d w|<B /|w|^{2}
$$

where $B$ is a constant; and if

$$
f(z)=e^{z} \int_{\alpha-i \infty}^{\alpha+i \infty} z^{-s} \Gamma(s) P(s) d s
$$

where $\alpha$ is a positive constant, then, as $z \rightarrow \infty$ in $|z|<\pi / 2$, uniformly $f(z) \rightarrow 0$.

Proof. To see that $f(z)$ is independent of $\alpha$, it is sufficient to show that

$$
J(y)=\int_{b}^{c} z^{-x-i y} \Gamma(x+i y) P(x+i y) d x \quad(0<b<c)
$$

approaches zero as $|y| \rightarrow \infty$. It is well known that

(82) $|\Gamma(x+i y)|=(2 \pi)^{1 / 2}\left[x^{2}+y^{2}\right]^{(x-1 / 2) / 2}(1+\eta) \exp \left\{-x-y \tan ^{-1}(y / x)\right\}$,

see F, p. 61 , where $\eta \rightarrow 0$ uniformly in $y$ as $x \rightarrow \infty$. Also $\eta \rightarrow 0$ uniformly in $x$ as $y \rightarrow \infty, x>0$. Utilizing (80) and (82) and setting $z=2 \rho^{2} e^{i \phi}$, we have

$$
\begin{aligned}
|J(y)|<\frac{6 B}{|y|} \int_{b}^{c} \exp \left\{|\phi y|-x \log 2 \rho^{2}-x-y \tan ^{-1} \frac{y}{x}\right. & \left.+\frac{2 x-1}{4} \log \left(x^{2}+y^{2}\right)\right\} d x \\
< & \frac{6 B}{|y|} \int_{b}^{c} \exp \left\{|\phi y|-b \log 2 \rho^{2}-b-y \tan ^{-1} \frac{y}{c}\right. \\
& \left.\quad+\frac{c}{2} \log \left(c^{2}+y^{2}\right)\right\} d x \\
& <\frac{6 B(c-b)}{|y|} \exp \left\{|y|\left[|\phi|-\tan ^{-1} \frac{y}{c}+\frac{c \log \left(c^{2}+y^{2}\right)}{2|y|}\right]\right\} .
\end{aligned}
$$


As $|y| \rightarrow \infty,\left(|\phi|-\tan ^{-1}(|y| / c)\right)$ becomes and remains negative for any fixed $\phi,|\phi|<\pi / 2$; therefore the exponential in the last member of (83) is bounded and $|\Im(y)| \rightarrow 0$.

Next select $\alpha$ so that the vertical path of integration for (81) passes through $s=z$ and its conjugate $s=\bar{z}$. Then holding the two points $z$ and $\bar{z}$ fixed, deform continuously the portion of the path between $z$ and $\bar{z}$ into a circular arc with center at the origin $s=0$ to get a new path which will facilitate the analysis.

To integrate (81) along this arc set $z=\rho e^{i \phi}, s=\rho e^{i \theta}$, and temporarily restrict $\phi$ so that $0 \leqq \phi<\pi / 2$; then the contribution of this integration to the value of $f(z)$ takes the form

$$
f_{1}(z)=\rho i \int_{-\phi}^{\phi} \Gamma\left(\rho e^{i \theta}\right) P\left(\rho e^{i \theta}\right) \exp \left\{i \theta+\rho e^{i \phi}-\rho e^{i \theta}[\log \rho+i \phi]\right\} d \theta .
$$

Again utilizing (80) and (82)

$$
\left|P\left(\rho e^{i \theta}\right) \Gamma\left(\rho e^{i \theta}\right)\right|<(6 B / \rho) \exp \{(\rho \cos \theta-1 / 2) \log \rho-\rho \cos \theta-\rho \theta \sin \theta\}
$$

and hence

$$
\left|f_{1}(z)\right|<\left(6 B / \rho^{1 / 2}\right) \int_{-\phi}^{\phi} \exp \{\rho[(\cos \phi-\cos \theta)+(\phi-\theta) \sin \theta]\} d \theta .
$$

Let $Q(\theta)=\cos \phi-\cos \theta+(\phi-\theta) \sin \theta$; then $Q(\phi)=0$ and $Q(-\phi)=-2 \phi \sin \phi$ $\leqq 0$. Over the entire range of integration $Q(\theta) \leqq 0$, because $d Q / d \theta=(\phi-\theta) \cos \theta$ $\geqq 0$. As a consequence $\left|f_{1}(z)\right|<6 \pi B / \rho^{1 / 2}$ and $\left|f_{1}(z)\right| \rightarrow 0$ as $\rho \rightarrow \infty$. When $-\pi / 2<\phi \leqq 0$, the same conclusion is reached in a similar way by adjusting a few signs.

Still assuming $0 \leqq \phi<\pi / 2$, consider next the contribution $f_{2}(z)$ to the value of $f(z)$ by the integration over the vertical path running from $z$ straight up to $(z+i \infty)$. To do this shift the origin to the point $z$ by setting $s=z+w$; then $f(z)$ takes the form

$$
f(z)=(2 \pi / z)^{1 / 2} \int(1+w / z)^{-1 / 2} \Phi_{1}(w+z) \exp \left\{w^{2} q(w / z) / 2 z\right\} d w
$$

where $\Phi_{1}(w+z)=P(w+z) / \Omega(w+z)$. Set $z=2 \rho^{2} e^{i \phi}$ and $w=2 i \rho \sigma$, then the contribution $f_{2}(z)$ becomes

$$
f_{2}(z)=\int_{0}^{\rho}+\int_{\rho}^{\infty} \frac{2 i \pi^{1 / 2} \Phi_{1}\left(2 \sigma \rho i+2 \rho^{2} e^{i \phi}\right) \exp \left\{-\sigma^{2} e^{-i \phi} q\left(\sigma i / \rho e^{i \phi}\right)\right\}}{e^{i \phi / 2}\left(1+i \sigma / \rho e^{i \phi}\right)^{1 / 2}} d \sigma .
$$

The following bounds are needed to estimate the size of $f_{2}$ and $f_{3}$ on the range $0 \leqq \phi<\pi / 2$ :

$$
\left|1 \pm i \sigma e^{\mp i \phi} / \rho\right|^{-1 / 2} \leqq 1 \quad(\rho>0, \sigma \geqq 0) ;
$$




$$
\begin{aligned}
& \left|1+i \sigma / \rho e^{i \phi}\right|^{-1 / 2}<(\rho / \sigma)^{1 / 2} \quad(\rho>0, \sigma>0) ; \\
& \left|\Phi_{1}\left( \pm 2 \sigma \rho i+2 \rho^{2} e^{ \pm i \phi}\right)\right|<B / \rho^{2} \quad(\rho>1, \sigma>0) \text {; } \\
& \left|\Phi_{1}\left( \pm 2 \sigma \rho i+2 \rho^{2} e^{ \pm i \phi}\right)\right|<B / 2 \sigma \rho \quad(\rho>1, \sigma>0) \text {; }
\end{aligned}
$$

and

$$
\mid \exp \left\{-\sigma^{2} e^{\mp i \phi} q\left( \pm \sigma i / \rho e^{ \pm i \phi}\right) \mid \leqq 1 \quad(\rho>0, \sigma \geqq 0) .\right.
$$

Inequalities (85) and (86) are obviously correct; (87) and (88) are immediate consequences of $(80)$ and the properties of $\Omega(w)$, see F, p. 39 .

To verify (89) when the upper signs are used, let $\tau=\sigma / \rho$ and

$$
S(\tau, \phi)=\left(\tau^{2} / 2 e^{i \phi}\right) q\left(\tau i e^{-i \phi}\right)=-e^{i \phi}\left(1+\tau i e^{-i \phi}\right) \log \left(1+i \tau e^{-i \phi}\right)+i \tau .
$$

Then $\partial \Re[S(\tau, \phi)] / \partial \tau=\arg \left(1+i \tau e^{-i \phi}\right)$. But since $\Re[S(0, \phi)]=0$ and, $\partial \Re[S(\tau, \phi)] / \partial \tau \geqq 0$ when $\tau \geqq 0, \Re[S(\tau, \phi)] \geqq 0$ and likewise $\Re\left[e^{-i \phi} q\left(\sigma i / \rho e^{i \phi}\right)\right]$ $\geqq 0$ and hence (89) is valid for the upper signs. The inequality can be verified in a similar way for the lower signs.

Return to (84) and use these bounds to estimate the first integration, with the result that

$$
\left|\int_{0}^{\rho} \cdots d \sigma\right|<6 \int_{0}^{\rho} \frac{B d \sigma}{\rho^{2}}=6 B / \rho .
$$

As for the second integration,

$$
\left|\int_{\rho}^{\infty} \cdots d \sigma\right|<6 \int_{\rho}^{\infty}(\rho / \sigma)^{1 / 2}(B / 2 \rho \sigma) d \sigma=6 B / \rho ;
$$

hence $\left|f_{2}(z)\right|<12 B / \rho$ and $\left|f_{2}(z)\right| \rightarrow 0$ uniformly as $\rho \rightarrow \infty, 0 \leqq \phi<\pi / 2$. When $-\pi / 2<\phi \leqq 0$, the same conclusion is reached in a similar way by adjusting a few signs and making other minor alterations.

To get the contribution $f_{3}(z)$ to $f(z)$ arising from the integration from $(\bar{z}-i \infty)$ to $\bar{z}$, shift the origin to $\bar{z}$ by setting $s=w+\bar{z}$ so that

$$
f(z)=e^{z} \int z^{-w-\bar{z}} \Gamma(w+\bar{z}) P(w+\bar{z}) d w .
$$

Then set $z=2 \rho^{2} e^{i \phi}, w=-2 i \sigma \rho$, and the contribution

$$
\begin{aligned}
f_{3}(z)= & \int_{0}^{\rho}+\int_{\rho}^{\infty} 2 i \pi^{1 / 2} e^{i \phi / 2}\left(1-i \sigma e^{i \phi} / \rho\right)^{-1 / 2} \Phi_{1}\left(-2 i \rho \sigma+2 \rho^{2} e^{-i \phi}\right) \\
& \cdot \exp \left\{-\sigma^{2} e^{i \phi} q\left(-i \sigma e^{i \phi} / \rho\right)+4 i \rho^{2} \sin \phi-4 \phi \rho \sigma\right. \\
& \left.-4 i \phi \rho^{2} \cos \phi-4 \phi \rho^{2} \sin \phi\right\} d \sigma .
\end{aligned}
$$

Again restricting $\phi$ to the range $0 \leqq \phi<\pi / 2$, using (87) and (89), and estimating the result of the first integration in (90) 


$$
\left|\int_{0}^{\rho} \cdots d \sigma\right|<6 \int_{0}^{\rho}\left(B / \rho^{2}\right) d \sigma=6 B / \rho .
$$

As to the second integration,

$$
\left|\int_{\rho}^{\infty} \cdots d \sigma\right|<\sigma \int_{\rho}^{\infty}(\rho / \sigma)^{1 / 2}(B / 2 \rho \sigma) d \sigma=6 B / \rho
$$

hence $\left|f_{3}(z)\right|<12 B / \rho$ and $f_{3}(z) \rightarrow 0$ uniformly as $\rho \rightarrow \infty, 0 \leqq \phi<\pi / 2$. Thus Theorem II is correct if $0 \leqq \phi<\pi / 2$. The same conclusion is reached if $-\pi / 2$ $<\phi<0$ by making appropriate minor changes in the analysis; therefore Theorem II is proved.

LEмma. If $|\phi| \leqq \pi / 2$, then as $b \rightarrow \infty$ the integral

$$
\Im(b)=\int_{0}^{b} \exp \left\{-\sigma^{2} e^{i \phi}+i \phi / 2\right\} d \sigma \rightarrow \pi^{1 / 2} / 2
$$

uniformly in $\phi$.

Proof. If $\phi=0$ or $\pm \pi / 2$, the limit is $\pi^{1 / 2} / 2$, as is well known, see F, p. 60 . If $|\phi| \leqq \pi / 4$, integrate $\int \exp \left\{-w^{2}\right\} d w$ first along the ray running from the origin $w=0$ to $w=b \exp \{i \phi / 2\}$, next along the arc $|w|=b$ from $w=b$ exp $\{i \phi / 2\}$ to $w=b$, and then from $w=b$ out along the real axis to infinity with the result that

$$
J(b)=\int_{0}^{\phi}(b i / 2) \exp \left\{-b^{2} e^{i \theta}+i \theta / 2\right\} d \theta-\int_{b}^{\infty} \exp \left\{-\sigma^{2}\right\} d \sigma+\pi^{1 / 2} / 2 .
$$

If $b>1, \int_{0}^{\infty} \exp \left\{-\sigma^{2}\right\} d \sigma<\int_{0}^{\infty} e^{-\sigma} d \sigma=e^{-b}$, and

$$
\left|\int_{0}^{\phi} b \exp \left\{-b^{2} e^{i \theta}+i \theta / 2\right\} d \theta\right|<b \int_{0}^{\pi / 4} \exp \left\{-b^{2} / 2^{1 / 2}\right\} d \theta<1 / b .
$$

These bounds make the lemma obviously correct if $|\phi| \leqq \pi / 4$.

If $\pi / 4<\phi \leqq \pi / 2$, let $w=x+i y$ and introduce the curvilinear coordinates $u$ and $v$ with $u=x^{2}-y^{2}, v=2 x y$. In the first quadrant draw the hyperbola $x y=c$ where $c=\left(b^{2} \sin \phi\right) / 2$. Let the respective rays $y=x \tan (\phi / 2)$ and $y=x$ intersect this hyperbola in points $A$ and $B$. Integrate $\int \exp \left\{-w^{2}\right\} d w$ along the first ray from the origin $w=0$ to $A$, then along the hyperbola from $A$ to $B$, and finally along the second ray $y=x$ from $B$ to infinity with the result that

$$
\int_{0}^{A} \exp \left\{-w^{2}\right\} d w=\pi^{1 / 2} / 2-\int_{A}^{B} \exp \left\{-w^{2}\right\} d w-\int_{B}^{\infty} \exp \left\{-w^{2}\right\} d w .
$$

Along the hyperbola

$$
\int_{A}^{B} \exp \left\{-w^{2}\right\} d w=\int_{b^{2} \cos \phi}^{0} \frac{\left(x^{2}-c i\right) \exp \{-u-2 i c\}}{2 x\left(x^{2}+y^{2}\right)} d u .
$$


As $w$ moves from $A$ to $B$ on $x y=c, x \geqq c^{1 / 2}$ and $\left|x^{2}-c i\right| / 2\left(x^{2}+y^{2}\right)<1$. Hence

$$
\left|\int_{A}^{B} \exp \left\{-w^{2}\right\} d w\right|<\int_{0}^{\infty} e^{-u} c^{-.5} d u=2^{1 / 2} / b(\sin \phi)^{1 / 2} .
$$

Along the ray $y=x$

$$
\int_{B}^{\infty} \exp \left\{-w^{2}\right\} d w=e^{\pi i / 4} \int_{b(\sin \phi)^{1 / 2}}^{\infty} \exp \left\{-i \sigma^{2}\right\} d \sigma .
$$

Since $1 / 2^{1 / 2} \leqq \sin \phi \leqq 1$, the bound in (91) and the integral in (92) uniformly approach zero as $b \rightarrow 0$; and therefore the lemma is correct if $\pi / 4<\phi \leqq \pi / 2$. By changing a few signs the same conclusion can be reached if $-\pi / 2 \leqq \phi$ $<-\pi / 4$ and the lemma is proved.

Theorem III. If $P(W)$ is a single-valued function of the complex variable $W=x+i y$; if save for a possible pole of the first order at the origin, $P(W)$ is analytic throughout a half-plane $x>x_{0}$, where $x_{0}$ is a fixed negative number; if uniformly

$$
P(W) \rightarrow c, \quad \text { a constant, as } W \rightarrow \infty
$$

in the sector $|\arg W| \leqq \pi / 2$; and if when $x>x_{0}$ and $|W| \geqq 1$, the

$$
\left|W \frac{d P(W)}{d W}\right|<B
$$

a constant;

then, as $z \rightarrow \infty$ in the sector $|\arg (z)| \leqq \pi / 2$, uniformly

$$
I(z)=e^{-z} \int_{0}^{\infty}\left[P(x) z^{x-1} / \Gamma(x)\right] d x \rightarrow c .
$$

Proof. In the proof details are omitted when the analysis closely follows F's work, care being taken to get a uniform bound as each estimate is made. The details are given however when the analysis deviates appreciably from $F$.

The theorem calls for integration along the real axis of the $W$-plane. It is advantageous to shift the path of integration; and starting at $W=0$ to run first directly out to the point $W=z$ and then to run to the right, parallel to the real axis, out to infinity. This change of path is readily justified by using (82) and (93).

The origin is shifted to $W=z$ by making the change of variable $x=w+z$, then

$$
I(z)=\int\left[e^{-z} P(w+z) z^{w+z-1} / \Gamma(w+z)\right] d w,
$$

where the path of integration in the new $w$-plane runs from $w=-z$ directly to $w=0$, and then out along the real axis of the $w$-plane to $+\infty$. Using F's 
notation, see pp. 38-42,

$$
I(z)=(2 \pi z)^{-.5} \int(1+w / z)^{1 / 2} \Phi(w+z) \exp \left\{-w^{2} q(w / z) / 2 z\right\} d w
$$

where $\Phi(w)=P(w) \Omega(w)$ with

$$
\Omega(w)=(2 \pi)^{1 / 2} e^{-w} w^{w-.5} / \Gamma(w)
$$

and,

$$
q(w)=2[(1+w) \log (1+w)-w] / w^{2} .
$$

Let $z=2 \rho^{2} e^{i \phi}$ and let $\sigma_{0}=\sigma_{0}(\epsilon)$ be a certain fixed positive number less than $(\rho-1), \rho$ large; then on the path of integration mark the seven points $w=-z,-z+e^{i \phi} / 2,-2 \rho \sigma_{0} e^{i \phi}, 0,2 \rho \sigma_{0}, 2 \rho^{2}$, and $w=\infty$. These points divide the path into six successive intervals. Integration over the first interval is indicated by $I_{1}$, over the second by $I_{2}$, and so on, so that $I(z)=I_{1}(z)+I_{2}(z)$ $+\cdots+I_{6}(z)$. More precisely

$$
\begin{aligned}
& I_{1}=\int_{0}^{.5}\left[P\left(\sigma e^{i \phi}\right) z^{\sigma \exp \{i \phi\}} e^{i \phi-z} / z \Gamma\left(\sigma e^{i \phi}\right)\right] d \sigma ; \\
& I_{2}=\int_{-\rho+1 / 4 \rho}^{-\sigma 0} \pi^{-.5} e^{i \phi / 2}(1+\sigma / \rho)^{.5} \Phi\left(2 \rho \sigma e^{i \phi}+2 \rho^{2} e^{i \phi}\right) \exp \left\{-\sigma^{2} e^{i \phi} q(\sigma / \rho)\right\} d \sigma ;
\end{aligned}
$$

$I_{3}$ has the same integrand as $I_{2}$ while the limits run from $-\sigma_{0}$ to 0 ;

$$
I_{4}=\int_{0}^{\sigma 0} \pi^{-.5} e^{-i \phi / 2}\left(1+\sigma e^{-i \phi} / \rho\right)^{1 / 2} \Phi\left(2 \rho \sigma+2 \rho^{2} e^{i \phi}\right) \exp \left\{-\sigma^{2} e^{-i \phi} q\left(\sigma e^{-i \phi} / \rho\right)\right\} d \sigma ;
$$

$I_{5}$ and $I_{6}$ have the same integrand as $I_{4}$ while the limits run respectively from $\sigma_{0}$ to $\rho$ and from $\rho$ to $\infty$.

If, for any given $\epsilon>0$, there exists a $\sigma_{0}=\sigma_{0}(\epsilon)>1$ sufficiently large, such that

$$
\begin{aligned}
& \left|I_{2}(z)\right|<\epsilon / 8 \\
& \left|I_{5}(z)\right|<\epsilon / 8
\end{aligned}
$$

and

$$
\left|\left(c e^{-i \phi / 2} / \pi^{1 / 2}\right) \int_{0}^{\infty} \exp \left\{-\sigma^{2} e^{-i \phi}\right\} d \sigma\right|<\epsilon / 8
$$

for all $\rho>\sigma_{0}+1$; and if, with $\sigma_{0}$ fixed, there exists a constant $\rho_{0}>\sigma_{0}+1$ such that for all $\rho \geqq \rho_{0}$

$$
\begin{aligned}
& \left|I_{1}(z)\right|<\epsilon / 8 \\
& \left|I_{6}(z)\right|<\epsilon / 8
\end{aligned}
$$




$$
\left|I_{4}(z)-\left(e^{-i \phi / 2} / \pi^{1 / 2}\right) \int_{0}^{\sigma_{0}} c \exp \left\{-\sigma^{2} e^{-i \phi}\right\} d \sigma\right|<\epsilon / 8
$$

and

$$
\left|I_{3}(z)-\left(e^{i \phi / 2} / \pi^{1 / 2}\right) \int_{-\sigma_{0}}^{0} c \exp \left\{-\sigma^{2} e^{i \phi}\right\} d \sigma\right|<\epsilon / 8,
$$

then $|I(z)-c|<\epsilon$ and Theorem III is proved. The existence of $\sigma_{0}$ and $\rho_{0}$ will now be established.

Condition (97) can be satisfied by selecting $\sigma_{0}$ sufficiently large by virtue of the lemma. To estimate $I_{5}(z)$ let

$$
\Psi=\left(1+\sigma / \rho e^{i \phi}\right)^{1 / 2} \Phi\left(2 \rho \sigma+2 \rho^{2} e^{i \phi}\right) ; \quad R=q\left(\sigma / \rho e^{i \phi}\right) ;
$$

and $\lambda(\rho, \sigma)=\sigma / \rho \log \left(1+\sigma / \rho e^{i \phi}\right)$; and proceed as in F, pp. 50-54 where he estimates the magnitude of his expression (53). It is then concluded that $\left|I_{5}(z)\right|<B / \sigma_{0}$ where $B$ is an appropriate constant. Hence $\left|I_{5}\right|$ can be made as small as desired by taking $\sigma_{0}$ sufficiently large.

In reaching this conclusion, instead of F's inequality (c), p. 51; we need the inequality

$$
\left|\exp \left\{-\sigma^{2} e^{-i \phi} q\left(\sigma / \rho e^{i \phi}\right)\right\}\right| \leqq 1 \quad(0<\sigma \leqq \rho) .
$$

To verify (102) observe that the real part of $e^{-i \phi} q\left(x e^{-i \phi}\right)$ has the same sign as the $\Re\left[x^{2} e^{-i \phi} q\left(x e^{-i \phi}\right)\right], x>0$. Moreover

$$
\frac{\partial}{\partial x} \Re\left[x^{2} e^{-i \phi} q\left(x e^{-i \phi}\right)\right]=2 \log \left|1+x e^{-i \phi}\right|>0, \quad x>0,|\phi| \leqq \pi / 2 .
$$

Also $q(0)=1$, because

$$
q(x)=2\left[\frac{1}{1 \cdot 2}-\frac{x}{2 \cdot 3}+\frac{x^{2}}{3 \cdot 4}-\cdots\right], \quad|x| \leqq 1 .
$$

Consequently the $\Re\left[x^{2} e^{-i \phi} q\left(x e^{-i \phi}\right)\right]>0, x>0$, and (102) is valid.

Also from (103) it is clear that if $-1<x<0, q(x)>1$ and hence

$$
\left|\exp \left\{-\sigma^{2} e^{i \phi} q(\sigma / \rho)\right\}\right| \leqq 1 \quad(\rho>0,-\rho<\sigma<0) .
$$

Inequality (104) is used when considering stipulation (95) with

$$
I_{2}(z)=\left(e^{i \phi} / \pi\right)^{1 / 2} \int_{-\rho+1 / 4 \rho}^{-\sigma_{0}} \Theta \exp \left\{-\sigma^{2} e^{i \phi} S\right\} d \sigma
$$

where $\Theta=(1+\sigma / \rho)^{1 / 2} \Phi\left(2 \sigma \rho e^{i \phi}+2 \rho^{2} e^{i \phi}\right)$ and $S=q(\sigma / \rho)$. An estimate on $\left|I_{2}\right|$ is procured in the same way that $\mathrm{F}$ treats his $K_{2}(\rho, \epsilon)$ on pp. 57-60 with the result that

$$
\left|I_{2}(z)\right|<B_{1} / \sigma_{0}+B_{2} / \sigma_{0} \log \sigma_{0}
$$


where the $B$ 's are appropriate constants. This completes half of the proof, for it is now evident that there exists a $\sigma_{0}$ satisfying (95), (96), and (97). With $\sigma_{0}$ fixed, turn next to the determination of $\rho_{0}$.

First consider the integral $I_{1}(z)$, and note that in the region $|w| \leqq 1 / 2$, $P(w) / \Gamma(w)$ is bounded, that is, $|P(w) / \Gamma(w)|<B$, a constant, because a possible singularity in $P(w)$ at the origin is counterbalanced by a pole of $\Gamma(w)$ at the origin. Hence, if $|\phi| \leqq \pi / 2$,

$$
\begin{aligned}
\left|I_{1}(z)\right| & <\exp \left\{-2 \rho^{2} \cos \phi\right\} \int_{0}^{1 / 2}\left(B / \rho^{2}\right) \exp \left\{\sigma \cos \phi \log 2 \rho^{2}-\phi \sigma \sin \phi\right\} d \sigma \\
& <\left(B / \rho^{2}\right) \int_{0}^{1 / 2} \exp \left\{.5 \log 2 \rho^{2}\right\} d \sigma<B / \rho
\end{aligned}
$$

and (98) can be satisfied by taking $\rho_{0}$ sufficiently large.

Turn now to $I_{4}(z)$ with $\sigma_{0}$ fixed and let $\rho \rightarrow \infty$, then uniformly $\left(1+\sigma / \rho e^{i \phi}\right)^{1 / 2}$ $\rightarrow 1 ; P\left(2 \rho+2 \rho^{2} e^{i \phi}\right) \rightarrow c ; \Omega\left(2 \rho \sigma+2 \rho^{2} e^{i \phi}\right) \rightarrow 1$, see F, p. 39 ; and $q\left(\sigma / \rho e^{i \phi}\right) \rightarrow 1$, see (103). Consequently

$$
I_{4}(z) \rightarrow\left(\pi e^{i \phi}\right)^{-1 / 2} \int_{0}^{\sigma_{0}} c \exp \left\{-\sigma^{2} e^{-i \phi}\right\} d \sigma .
$$

uniformly as $\rho \rightarrow \infty$ and (100) can be satisfied by taking $\rho_{0}$ sufficiently large.

Next consider $I_{3}$ with $\sigma_{0}$ fixed and let $\rho \rightarrow \infty$; then uniformly $(1+\sigma / \rho)^{1 / 2} \rightarrow 1$, $\Phi\left(2 \rho \sigma e^{i \phi}+2 \rho^{2} e^{i \phi}\right) \rightarrow c$, and $q(\sigma / \rho) \rightarrow 1$, see (103). Consequently

$$
I_{3}(z) \rightarrow\left(e^{i \phi} / \pi\right)^{1 / 2} \int_{-\sigma_{0}}^{0} c \exp \left\{-\sigma^{2} e^{i \phi}\right\} d \sigma
$$

uniformly as $\rho \rightarrow \infty$ with $|\phi| \leqq \pi / 2$ and so for sufficiently large $\rho$, say $\rho \geqq \rho_{0}$, (101) is satisfied.

Finally $\left|I_{6}(z)\right|$ is estimated in the same way that $\mathrm{F}$ handles his integral (54) on pp. 54-55; and it is found that

$$
\left|I_{6}(z)\right|<B \int_{\rho}^{\infty}(1+\sigma)^{1 / 2} \exp \{-\sigma / 5\} d \sigma
$$

where $B$ is an appropriate constant. This bound on $\left|I_{6}\right|$ is obviously less than $\epsilon / 8$ if $\rho$ is sufficiently large. There is however a crucial step in making this estimate of $\left|I_{6}\right|$; we need the inequality

$$
\left|\exp \left\{-\sigma^{2} e^{-i \phi} q\left(\sigma / \rho e^{i \phi}\right)\right\}\right|<\exp \{-\sigma / 5\} \quad(\sigma \geqq \rho>1) .
$$

To verify (105) it suffices to show that the real part of

$$
u(\eta, \phi)=\left(\sigma e^{-i \phi} / 2 \rho\right) q\left(\sigma e^{-i \phi} / \rho\right), \quad \eta=\sigma / \rho,
$$

exceeds $1 / 10$ when $\eta \geqq 1$ and $|\phi| \leqq \pi / 2$. Since 


$$
\frac{\partial}{\partial \eta} \Re[u(\eta, \phi)]=(1 / \eta) \Re\left[1-\left(\eta e^{-i \phi}\right)^{-1} \log \left(1+\eta e^{-i \phi}\right)\right]
$$

it follows from the fact that

$$
\begin{aligned}
& \frac{\log (1+z)}{z} \mid \leqq\left[\left(\log 2^{1 / 2}\right)^{2}+(\pi / 4)^{2}\right]^{1 / 2}=.858 \cdots \\
& \quad(|z| \geqq 1 ;|\arg (z)| \leqq \pi / 2),
\end{aligned}
$$

that

$$
\frac{\partial}{\partial \eta} \Re[u(\eta, \phi)]>0 .
$$

Therefore $\Re[u(\eta, \phi)]$ increases as $\eta$ increases, $\phi$ fixed.

To find the smallest value of $\Re[u(\eta, \phi)]$ we need consider then only the functions

$$
\begin{aligned}
p(\phi) & =\Re[u(1, \phi)]=\left(\cos ^{2} \phi / 2\right) \log \left(4 \cos ^{2} \phi / 2\right)-1+(\phi \sin \phi) / 2, \\
p^{\prime}(\phi) & =\sin \phi g(\phi) \quad \text { where } \quad g(\phi)=(\phi / 2) \cot \phi-\log (2 \cos \phi / 2)
\end{aligned}
$$

and $g^{\prime}(\phi)=\csc ^{2} \phi(\sin \phi-\phi) / 2$. The derivative $g^{\prime}(\phi) \leqq 0$ on the range $0 \leqq \phi \leqq \pi / 2$ and hence $g(\phi)$ decreases as $\phi$ increases. Since $g(0)=.5-\log 2=-.193 \cdots$, $g(\phi)<0$ and $p^{\prime}(\phi)<0$. But $p(\phi)$ is an even function and $p(\pi / 2)=.5 \log 2-1$ $+\pi / 4=.131 \ldots$; hence $(105)$ is correct. This completes the proof of Theorem III.

\section{BIBLIOGRAPHY}

1. G. Doetsch, Theorie und Anwendung der Laplace-Transformation, 1943, p. 116.

2. W. B. Ford, The asymptotic developments of functions defined by Maclaurin series, University of Michigan, Science Series, vol. 11, 1936.

3. E. R. Heineman, Trans. Amer. Math. Soc. vol. 31 (1929) pp. 464-476.

4. H. K. Hughes, Bull. Amer. Math. Soc. vol. 50 (1944) pp. 425-430.

5. — Bull. Amer. Math. Soc. vol. 51 (1945) pp. 456-461.

6. C. V. Newsom, Amer. J. Math. vol. 60 (1938) pp. 561-572.

7. H. Scheffé, Trans. Amer. Math. Soc. vol. 40 (1936) pp. 127-154.

8. W. J. Trjitzinsky, Acta Math. vol. 62 (1934) pp. 167-226.

9. E. M. Wright, Philos. Trans. Roy. Soc. London Ser. A. vol. 238 (1940) pp. 423-451.

10. —_, Trans. Amer. Math. Soc. vol. 64 (1948) pp. 409-438.

11. - - J. London Math. Soc. vol. 10 (1935) pp. 286-293.

12. _-—, Proc. London Math. Soc. (2) vol. 46 (1940) pp. 389-408.

The University of Minnesota, Minneapolis, MinN. 